\title{
An Energy-Efficient Proactive Routing Scheme for MANET: Game Theoretical Approach of Forwarding with Selfish Nodes
}

\author{
Abdelkabir Sahnoun, Ahmed Habbani, and Jamal El Abbadi
}

\begin{abstract}
In Mobile Ad-hoc Networks, nodes exchange packets with each other using intermediate nodes as relays. Since nodes in MANETs are battery powered, energy conservation is a crucial issue. Accepting relay all request may not be in the best interest of a node. But if many nodes prefer not to consume energy in relaying packets on behalf of others, the overall performance of routing in network will be influenced. In this paper we address the energyefficient routing problem in MANETs with selfish nodes. We modeled this problem as a game-theoretic constraint optimization; we defined the utility of each node as a weighted difference between a performance metric and some transmission costs. A motivate mechanism is proposed in order to induce nodes to forwarding cooperation. Each node independently implements the optimal equilibrium strategy under the given constraints. Simulation results by NS3 simulator show that our proposed approach can improve system performance in network lifetime and packet delivery ratio.
\end{abstract}

\section{Keywords - Game theory, MANETs, Energy-Efficiency}

\section{INTRODUCTION}

A Mobile Ad Hoc Network (MANET) is a collection of wireless mobile nodes that out of necessity form a network required to facilitate communication. The network does not have infrastructure per say, and the nodes freely move. Each node must work as a router and forward messages to the appropriate device. Since mobile ad hoc networks change their topology frequently without prior notice, routing in such networks is a challenging task, in recent years, many routing protocols have been proposed for MANETs. These protocols can be classified into three different groups: proactive, reactive and hybrid. In proactive routing protocols such as DSDV [1] and OLSR [2], the routes to all the destination (or parts of the network) are determined at the start up, and maintained by using a periodic route update process. In reactive protocols such as AODV [3] and DSR [4] routes are determined when they are required by the source using a route discovery process. Hybrid routing protocols combines the basic properties of the first two classes of protocols into one. In particular, energy efficient routing may be the most important design criteria for MANETs, since mobile nodes will be powered by batteries with limited capacity. Power failure of a mobile node not only affects the node itself but also its ability to forward packets on behalf of others and thus the overall network lifetime. Simple routing schemes are sufficient only if all nodes are willing to participate in the forwarding. However, nodes are energy constrained by

A. Sahnoun and J. El Abbadi are with LEC Laboratory, EMI, Mohammed V University, Rabat, Morocco (e-mail: abdelkabirsahnoun@ @research.emi.ac.ma, elabbadi@emi.ac.ma).

A. Habbani is with SIME Laboratory, ENSIAS, Mohammed V University, Rabat, Morocco (e-mail: habbani@ifride.com). their battery level and want to maximize their lifetime, which leads to potential selfishness as the nodes may refuse to forward packets for other nodes. Forwarding for other nodes' packets consumes a node's limited energy. Therefore a node has no interest in forwarding packets on behalf of another node, thus if many of the nodes act selfishly, few multi-hop communications can take place and the network functionality is compromised. Hence, it is necessary to design a mechanism to enforce cooperation for packet forwarding among greedy and distributed nodes.

In order to overcome this problem, several authors have recently proposed stimulating cooperation using incentives, to enforce cooperation for packet forwarding among nodes. Most of the approaches proposed in the literature, such as those presented in [5] and [6], are focused on the packet forwarding phase of a routing protocol, relatively little attention has been devoted to the problem of stimulating cooperation in the route discovery phase of a routing protocol. We investigate that a suitable tool for modeling the routing behaviors in MANETs with nodes' selfishness is game theory [7].

In this paper, we define a non-cooperative game theoretic model to support more energy-aware routing in MANETs under the constraint of nodes' selfishness. The proposed model uses local information. So, it is applicable in MANETs. We integrate this model to OLSR protocol that is an optimization over the classical link state protocol for the MANETs. Simulation results show that our proposed model improves.

The remainder of this paper is organized as follows. In section 2 we shortly describe the related work. Section 3 presents brief review of OLSR routing protocol. Section 4 discusses our proposed routing scheme. The performance of this approach is evaluated by extensive simulation; which is presented in Section 5. Finally the conclusion remarks are given in Section 6.

\section{RELATED WORK}

The problem of obtaining cooperative routing behavior in wireless ad hoc networks consisting of inherently selfish nodes has received considerable attention in recent years. Therefore, many protocols and algorithms have been proposed to circumvent this problem. As nodes in MANETs are generally battery-operated, to maximize the energy efficiency as much as possible, the first attribute that has to be conserved is energy consumption. As a consequence, plenty of research efforts in energy-aware routing for MANETs become predominant. In [8] Jung and Hundewale have applied new energy efficiency metrics to MANET routing protocol, they used adaptive load balancing model. Yet they haven't uncovered sufficient detail about how to fulfill energy load balancing in mobile ad-hoc networks. In [9] Feeney has presented a technique for evaluating the consumption energy manner in mobile ad-hoc networks, this 
technique was used to investigate the energy consumption of few MANET routing protocol. In [10] Khouzani and Eshghi have examined the purpose of epidemic routing in energy constrained DTN. They argued that dynamic optimal strategies simulate some threshold-based principles.

Several authors have proposed stimulating cooperation, to handle the problem of routing in MANETs with selfish nodes, using incentives. For instance, in [11] Manam and Mahendran have investigated an analytical method to evaluate the effectiveness of some routing protocols considering that consists of selfish nodes that have different ranges of transmission. However Wang and Singhal in [12] have proposed a novel routing algorithm for MANETs with selfish nodes. They present a protocol using a motivating mechanism to promote cooperation among nodes, their proposition concentrate on the truthfulness. Watchdog and pathrater are proposed in [13] to identify misbehaving nodes and deflect traffic around them. Reputation-based protocols are proposed in [14] and [15]. In [16] a novel mechanism has proposed to present cooperation among nodes. In [17] Felegyhazi and Hubaux have used game theory and graph theory to prove that cooperation for packet forwarding can be implemented without incentive mechanisms. In [18] Srinivasan and Nuggehalli have proposed another type of game theory called tit for tat, to overcome selfishness problem in packet forwarding. However in [19] Srinivasan, Nuggehalli, Chiasserini, and Rao have applied game theory to get a distributed algorithm based on which nodes decide whether or not to accept a relay request. Naserian and Tepein [20] have proposed a routing protocol based on forwarding game for MANET. In their protocol, a node enters the forwarding game upon receiving a flooding packet. Parameters such as residual energy level, channel congestion, number of packets in the node's transmission queue, and the distance from the source of the flooding packet are included for computing utility.

In our work, we have modeled a forwarding cooperation for Multi Point Relay selection algorithm in OLSR routing protocol, by adopting an incentive mechanism and a noncooperative game theory. We have illustrated that our algorithm leads to Nash equilibrium, which can be used to improve energy efficiency in MANETs routing.

\section{BRIEF REVIEW OF OLSR ROUTING PROTOCOL}

OLSR (Optimized Link State Routing), [2], is a proactive routing protocol where nodes periodically exchange topology information in order to establish a route to any destination in the network. It is an optimization of a pure link state routing protocol, based on the concept of multipoint relays (MPRs). First, using multipoint relays reduces the size of the control messages: rather than declaring all its links in the network, a node declares only the set of links with its neighbors that have selected it as "multipoint relay". The use of MPRs also minimizes flooding of control traffic (fig. 1). Indeed only multipoint relays forward control messages. This technique significantly reduces the number of retransmissions of broadcast messages. OLSR consists of two main functionalities:

-Neighborhood discovery. Each node acquires the knowledge of its one-hop and two-hop neighborhood by periodic Hello messages. It independently selects its own set of multipoint relays (MPRs), among its one-hop neighbors in such a way that its MPRs cover (in terms of radio range) all its two-hop neighbors.

-Topology dissemination. Each node also maintains topological information about the network obtained by TC (Topology Control) messages, broadcast by MPR nodes.

Each node computes its routing table by the Dijkstra algorithm. This table provides the shortest route (i.e. the route with the smallest hop number) to any destination in the network.

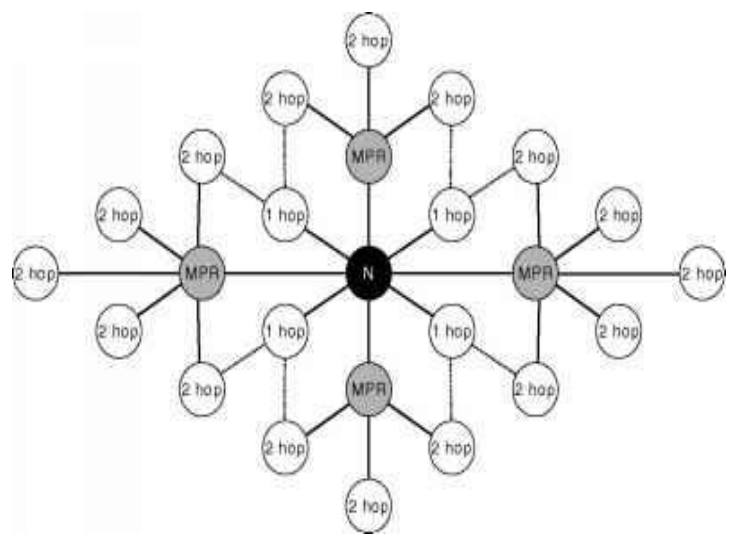

Fig 1. MPR Mechanism

\section{PROBLEM DEFINITION}

\section{A. Application Scenario and Motivation Mechanism}

An ad-hoc network is modeled as a graph $G(V, E)$ where $V$ represents the set of nodes and $E$ is the set of links. Nodes in the network have limited energy depending on the initial batterypower. In MANET routing each node generate packets representing many types of messages and send them to each other. A mobile node consumes its battery energy not only when it actively sends or receives packets, but also when it stays idle listening to the wireless medium for any possible communication requests from other nodes.

A wireless node's radio can be in one of the following four states: Transmit, Receive, listening or Sleep and each of which consumes different levels of energy.

- Transmit: when a node is transmitting a packet to another node with transmission energy $E_{T}$;

- Receive: when a node is receiving a packet from another node with reception energy $E_{R}$. The receiving node may be the destination node or just an intermediate;

- listening: Even when no messages are being transmitted over the medium, the nodes keep listening the medium with listening energy $E_{L}$;

- Sleep: when the radio is turned off and the node is not capable of detecting signals: no communication is possible.

The node spends sleep energy $E_{S}$ that is largely smaller than any other energy.

Generally any wireless network is following the above constraint:

$$
E_{T}>E_{R}>E_{L}>E_{S}
$$

In Mobile Ad-hoc Networks, in order to minimize the overhead from flooding of control traffic, it uses only selected nodes (MPR) to retransmit control messages. This technique significantly reduces the number of retransmissions required to flood a message to all nodes in the network. The neighbors of 
the transmitter which are not in its MPR set, receive and process broadcast messages but do not retransmit them. Maybe a node receives several messages from different transmitters at the same time. Then the receiver must determine which one to accept. Some message can be dropped, since each node has a limited data cache, which is used to store data packets for transmission.

Some nodes in MANET behave with selfishness, since they want to maximize their benefits with least cost to conserve their energies as much as possible during the routing process, which can affect the entire network if many nodes behave like that. To overcome this problem, we have proposed a motive mechanism using local information, combining between motivate retransmitters and punishing selfish node. We use a weightedbased evaluating scheme, where a weight called Participation Value $(P V)$ is assigned dynamically at each node, the algorithm initially assign a $P V$ equal to 0 to each node, then each time a node forward a packet for other nodes the $P V$ will be incremented, and each time the node refuse to forward a packet, by passing to sleep mode, the $P V$ will be decremented. If more than one transmitter sends packets to a receiver at the same time, the receiver will accept the packet from the one with larger $P V$. This approach indicates that nodes need to periodically receive other nodes $P V$. Thus it is crucial to find a way to propagate $P V$ nodes to the neighbors without increasing network overhead. A convenient way is to include this information to the Hello packets that are periodically generated by each node. Therefore we extend the Hello packet to contain field for the $P V$ of the sender node.

\section{B. Overview of Game Theory}

The purpose of game theory is a game which describes a formal model of an interactive situation. In describing a noncooperative game [21], the notion of a strategic form proves to be one of the most popular representations. In this regard, a noncooperative game in strategic has three components: the set of players, their strategies, and the payoffs or utilities. More formally, a strategic game is defined as follows:

Definition.1 A non-cooperative game in strategic form is a triplet $G=\left(N,\left(S_{i}\right)_{i \in N},\left(u_{i}\right)_{i \in N}\right)$, where:

- $N$ is a finite set of players, i.e., $N=\{1, \ldots, \mathrm{N}\}$.

- $S_{i}$ is the set of available strategies for player $i$.

- $u_{i}: S \rightarrow \mathbb{R}$ is the utility (payoff) function for player $i$, with $S=$ $S_{1} \times \cdots \times S_{i} \times \cdots S_{N}$.

For any player $i$, every element $s_{i} \in S_{i}$ is the strategy of $i$, $s_{-i}=\left[s_{j}\right]_{j \in N, j \neq i}$ denote the vector of strategies off all players except $i$, and $s=\left(s_{i}, s_{-i}\right) \in S$ is referred to as a strategy profile. Whenever the sets of strategies $S_{i}$ are finite for all $i \in N$, the game is called finite. For a game in strategic form, each player has to select a strategy so as to optimize its utility function. Whenever each player $i \in N$ selects a strategy $s_{i} \in S_{i}$ in a deterministic manner, i.e., with probability1, then this strategy is known as a pure strategy. In order to analyze a noncooperative game in strategic form, one must first clearly specify the players, their strategies, and their potential payoffs. In this context, any two-player non-cooperative finite game can be represented in a matrix format whereby the strategies of the players constitute the rows and columns of the matrix, and each element is a pair of numbers that represent the payoffs for the two players when a certain combination of strategies is used.
The majority of non-cooperative games are not solvable by iterated dominance, so alternative solution concepts must investigated. In this regard, the most accepted solution concept for a non-cooperative game is that of a Nash equilibrium, a Nash equilibrium is a state of a non-cooperative game where no player can improve its utility by changing its strategy, if the other players maintain their current strategies. The Nash equilibrium is defined as follows:

Definition.2 A pure-strategy Nash equilibrium of a noncooperative game $G=\left(N,\left(S_{i}\right)_{i \in N},\left(u_{i}\right)_{i \in N}\right)$ is a strategy profile $s^{*} \in S$ such that $\forall i \in N$ we have the following:

$$
u_{i}\left(s_{i}^{*}, s_{-i}^{*}\right) \geq u_{i}\left(s_{i}, s_{-i}^{*}\right), \forall s_{i} \in S_{i}
$$

In other words, a strategy profile is a pure-strategy Nash equilibrium if no player has an incentive to unilaterally deviate to another strategy, given that other players' strategies remain fixed.

\section{Game Modeling}

In this section we present a new framework that allows investigating the problem of energy-efficiency routing in OLSR by modeling it as a non-cooperative game, as each player in a non-cooperative game, the nodes attempt to maximize their benefits by taking the suitable actions. We adopt this game when broadcasting control messages using MPRs, the MPRs are intermediates nodes that consume energy to forward packets for their MPR selectors (nodes that select them as MPRs).

Let's consider a simple game between a transmitter node $n_{t}$ and a re-transmitter node $n_{r}$ that's one of its MPRs. The $n_{t}$ has to choose between two actions: either to select an MPR to retransmits it's control message or not, while the $n_{r}$ can accept to forward or reject by passing to sleep mode according to energetic requirements. We assume that time is slotted, and nodes will take their actions simultaneously; in each time slot, a node is only allowed to one of its actions. Each node select it's MPR set, in order to minimize the overhead of flooding messages in the network by reducing redundant retransmissions in the same region, so in this game, we consider this gain in energy as a benefit for this node (the transmitter).

The utility function for a given transmitter node $u\left(n_{t}\right)$ and a retransmitter one $u\left(n_{r}\right)$ in this game is simply given as follow:

To forward a broadcast message to the two-hop neighbors covered by an MPR node, if $n_{t}$ selects to do by means of MPR node $n_{r}$ and the latter being on active mode (accept to forward), the first and the second will spend the energy of transmission $E_{T}$ each, but we reward the $n_{r}$ with augmenting its $P V$ by the number of two-hop neighbors $n b_{2-h o p}$ covered by it.

$$
\begin{aligned}
& u\left(n_{t}\right)=-E_{T} \\
& u\left(n_{r}\right)=-E_{T} \text { and } P V \rightarrow P V+n b_{2-h o p}
\end{aligned}
$$

but if $n_{r}$ being on sleep mode (reject to forward), we decrement its $P V$ value by 1 , the $n_{r}$ will retransmit the massage again by means of more one-hope neighbors to reach all the two-hop neighbors $n b_{2-h o p}$ covered by this $n_{r}$, in this case we need at least a number of retransmission equal to $n b_{2-h o p}$. So the utility functions will be as follows.

$$
\begin{aligned}
& u\left(n_{t}\right)=-E_{T} \times\left(1+n b_{2-h o p}\right) \\
& u\left(n_{r}\right)=0 \text { and } P V \rightarrow P V-1
\end{aligned}
$$


In the second case for $n_{t}$, if it doesn't adopt $n_{r}$ as MPR and the latter being ready to forward, we need at least a number of retransmission equal to $n b_{2-h o p}$ directly, and the $n_{r}$ will spend the energy of listening $E_{L}$ correspondent to listening mode. So the utility functions will be as follows.

$$
\begin{aligned}
& u\left(n_{t}\right)=-E_{T} \times n b_{2-h o p} \\
& u\left(n_{r}\right)=-E_{L}
\end{aligned}
$$

Else if the $n_{r}$ being in sleep mode, we get the utility functions below.

$$
\begin{aligned}
& u\left(n_{t}\right)=-E_{T} \times n b_{2-h o p} \\
& u\left(n_{r}\right)=0 \text { and } P V \rightarrow P V-1
\end{aligned}
$$

The payoff matrix for the game is given in Table I.

TABLE I

PAYOFF MATRIX FOR THE GAME

\begin{tabular}{|l|l|l|}
\hline$n_{t}$ & \multicolumn{1}{|c|}{ Forward } & \multicolumn{1}{|c|}{ Sleep } \\
\hline Select & $\left(-E_{T},-E_{T}\right.$ and $\left.P V \rightarrow P V+n b_{2-h o p}\right)$ & $\left(-E_{T} \times\left(1+n b_{2-h o p}\right), 0\right.$ and $\left.P V \rightarrow P V-1\right)$ \\
\hline Don't select & $\left(-E_{T} \times n b_{2-h o p},-E_{L}\right)$ & $\left(-E_{T} \times n b_{2-h o p}, 0\right.$ and $\left.P V \rightarrow P V-1\right)$ \\
\hline
\end{tabular}

During the game, nodes will take different actions to maximize their benefit. The unique Nash Equilibrium in this game is that the $n_{t}$ broadcast control messages via $n_{r}$ as MPR and the latter retransmit. Nevertheless, a $n_{r}$ can base his decision on its energetic state or requirements, for example if its data cache is full in current slot, it won't be able to receive more packets, or it has a low residual energy, it would rather choose not to participate in routing. For this purpose, we propose an indicator taking in consideration both residual energy and the MAC queue, based on it, the node decides either to retransmit or not. We compute an energetic cost $E C_{i}$ and a MAC queue utilization cost $M Q U C_{i}$ for each node $\boldsymbol{i}$, as shown in Equations (7) and (8).

$$
\begin{aligned}
E C_{i} & =\frac{R E_{i}}{I E^{N}} \\
M Q U C_{i} & =\frac{P_{i}}{M Q_{\max }}
\end{aligned}
$$

Where $R E_{i}$ is the residual energy at each time, $I E$ is the initial energy of the node, $N P_{i}$ is the number of packet in the MAC queue and $M Q_{\text {max }}$ is the maximum considered MAC queue size.

The decision taken by the $n_{r}$ is based on its $E C_{i}$ and $M Q U C_{i}$ values, if $E C_{i}$ value is less than a given threshold $\alpha$ or $M Q U C_{i}$ value is greater than a given threshold $\beta, n_{r}$ would rather choose not to participate in routing. The acceptance algorithm is as follows.

- If $E C_{i}<\alpha$ or $M Q U C_{i}>\beta \quad$ Reject

- Else Accept.

\section{SIMULATION RESULTS}

In this section, we have simulated our modified version of OLSR using NS3 network simulator, to evaluate the performance of the proposed method. We considered three performance metrics to evaluate this approach, which are: Average Energy Consumption per node, Packet Lost Ratio, and Network Lifetime (the time until the battery of a mobile node depletes). We simulated a MANET with a variant number of nodes, within a $1500 \times 1500$ meter square region. There are 5 UDP sources generating packets of 1024 bytes, we simulated each scenario 5 times, mobile nodes move in the area based on a Random Waypoint mobility model with maximum speed of $15 \mathrm{~m} / \mathrm{sec}$, we took in this simulation $\alpha=0.15$ as a threshold for the residual energy cost and $\beta=0.95$ as a threshold for the MAC queue utilization cost. The simulation time is set to 400 seconds. We executed the simulations to evaluate the efficiency of our modified scheme compared to the standard OLSR. Simulation parameters are shown in Table II.

TABLE II

SIMULATION PARAMETERS

\begin{tabular}{cc}
\hline \hline Parameter & Value \\
\hline Area & $1500 \mathrm{~m} \times 1500 \mathrm{~m}$ \\
Nodes & $30,40,50$ \\
Traffic sources & 5 \\
Traffic Type & CBR / UDP \\
Packet Size & 1024 bytes \\
Start of Traffic & 50 seconds \\
Transmission Power & $7.5 \mathrm{dbm}$ \\
Link bandwidth & $2 \mathrm{Mb} / \mathrm{s}$ \\
Initial Node Energy & $4 \mathrm{Joules}$ \\
Simulations/Scenario & $5 \mathrm{times}$ \\
Nodes'speed & $15 \mathrm{~m} / \mathrm{sec}$ \\
$\alpha$ & 0.15 \\
$\beta$ & 0.95 \\
Simulation time & $400 \mathrm{~seconds}$ \\
\hline \hline
\end{tabular}

In a first simulation, we simulated a MANET with 40 nodes, we intended to evaluate our proposed algorithm, from energy consumption point of view, by comparing the average node energy consumption by time, between modified and standard OLSR. The purpose is to perform an energy analysis of the behavior of our modified scheme.

We present the results in Fig. 2. As shown in this figure, modified OLSR decreases energy consumption per node around $6.5 \%$ as average diminishing. Energy consumption is directly related to the size and number of control packets generated by nodes. Thus, this indicates that our modified scheme achieves more uniform utilization of network resources by adopting the residual energy cost and MAC queue utilization cost of each node, and dispersing control messages through different retransmitters to reduce energy consumption. Meanwhile the standard OLSR rebroadcasts traffic controls using same 
intermediate nodes, which results fast depletion of their battery powers that lead to more packet lost and then more retransmissions of control packets.

In a second setup, in order to examine whether our approach contributes to the increase of network lifetime, without loss of performance, we simulated a MANET with a variant number of nodes (30, 40, 50 nodes), we considered two other performance metrics: Packet Delivery Ratio (PDR) and Network Lifetime (in second). For network lifetime measurements, we execute the simulations until a node is completely depleted.

Figure 3 shows that the modified OLSR outperforms the standard OLSR in term of network lifetime. This indicates that our algorithm avoids intermediate nodes that have a low residual energy value, which extends a bit little the life time of these nodes. Even though the intermediate nodes that been chosen, maybe not consist the shortest path to the destination.

Figure 4, illustrates that as the number of nodes augments, a lot of packets will be lost due to network congestion, thus we notice a significant augmentation in Packet Lost Ratio for both protocols. But we observe a bit little improvement in the case of modified OLSR, due to the fact that our scheme takes in consideration the value of MAC queue utilization when deciding to receive more messages, so when a node has a full MAC queue, all packets sent to it, will be dropped, that are most likely to cause loss of packets.

We observe that as the number of nodes increase in the network, the improvement in both network lifetime and Packet delivery Ratio PDR decreases, due to the fact that number of packets moved in the network augments, which causes a lack of robustness of our method to adjust with highly changing dynamic environment.

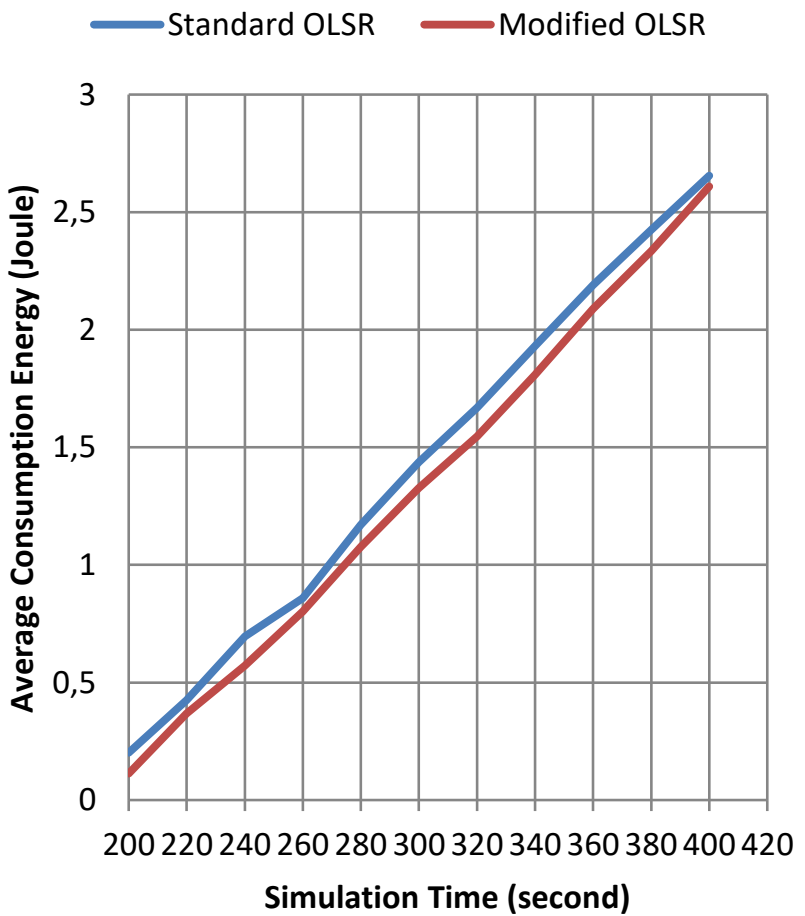

Fig. 2. Average Energy Consumption per Node

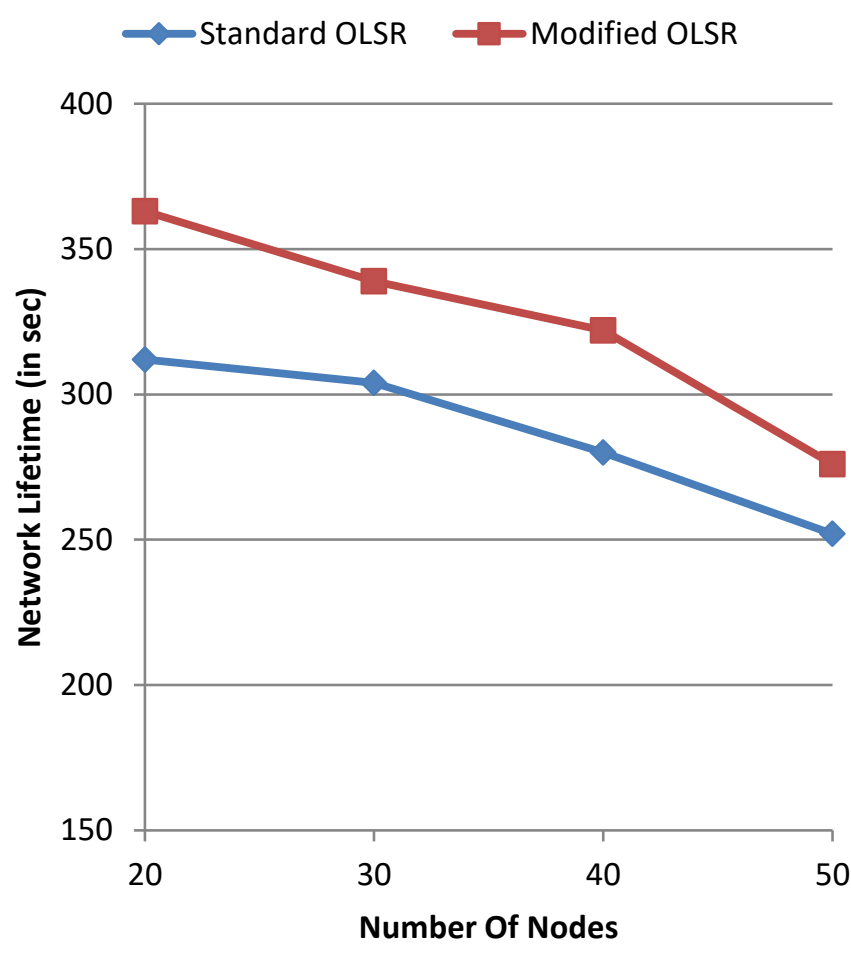

Fig. 3. Network Lifetime

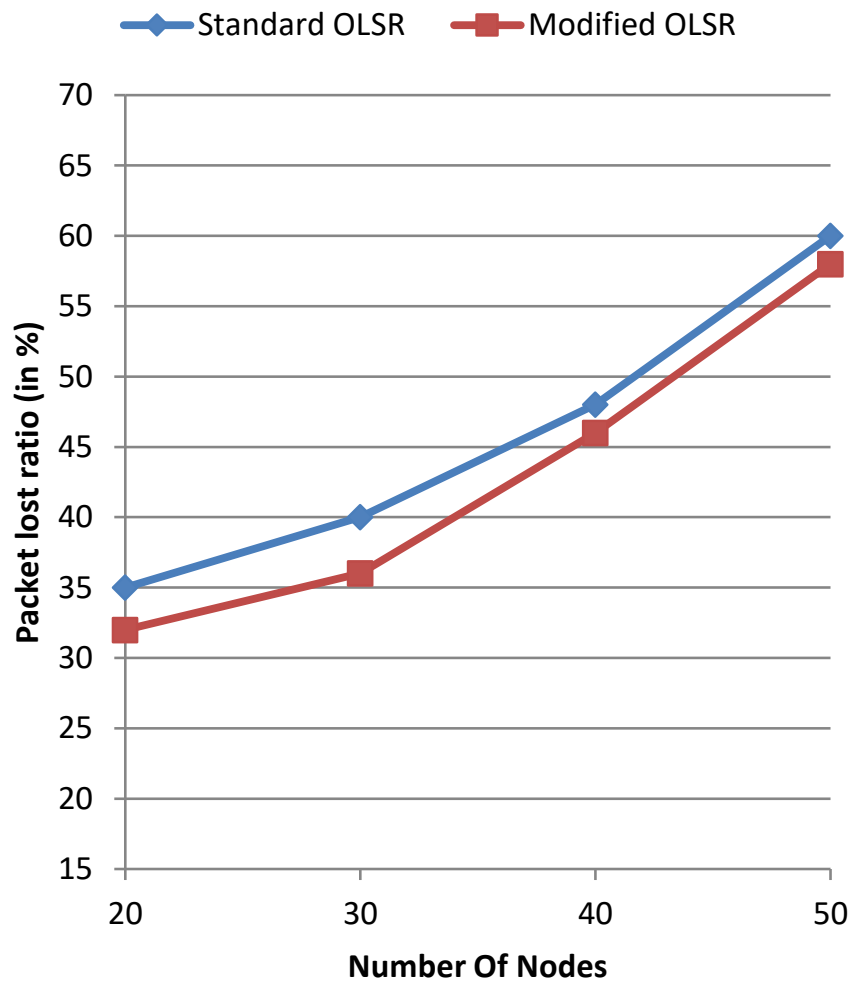

Fig. 4. Packet Lost Ratio. 


\section{CONCLUSIONS}

In MANETs, the lack of centralized control leads to the fact that the behavior of individual nodes has a profound effect on network performance. On of major examples of this problem, is when users reject to relay requests for the sake of energy resources, which presents a selfish behavior.

In this paper, we have introduced an incentive technique for inducing forwarding cooperation during routing packets. We have taken the proactive routing protocol OLSR as a case study. Then we have modeled the problem of routing with nodes' selfishness as a game-theoretic approach. We consider the competitive relationships between the transmitters and their neighbors in MANET as a simultaneous game and give the corresponding game theoretical formulation in detail. Moreover, one simple proposed solution is presented for the problem. Simulation results show that our method integrated in OLSR protocol reduces both energy consumption and packet lost ratio, and increases network lifetime compared with the standard OLSR.

Different extensions can be considered as future work. The most natural one is to find a way to modeling the relationships as another kind of game called: coalition game, which may give more potential solution for the nodes' selfishness problem, by using another type of cooperation among nodes in network.

\section{REFERENCES}

[1] C. E. Perkins and P. Bhagwat, "Highly dynamic Destination Sequenced Distance-Vector routing (DSDV) for mobile computers", In Proceedings of the SIGCOMM '94 Conference on Communications Architectures, Protocols and Applications, 1994, pp. 234-244.

[2] T. Clausen and P. Jacquet "Optimized Link State Routing Protocol (OLSR)." RFC 3626, IETF Network Working Group, October 2003.

[3] C. E. Perkins and E. M. Royer, "Ad Hoc On-demand Distance Vector Routing," In Proceedings of the 2nd IEEE Workshop on Mobile Computing Systems and Applications, 1999, pp. 90-100.

[4] D. B. Johnson and D. A. Maltz, "Dynamic Source Routing in Ad-Hoc Ad hoc Networks", Mobile Computing, 1996, pp. 153-181.

[5] K. Chen and K. Nahrstedt, "iPass: An Incentive Compatible Auction Scheme to Enable Packet Forwarding Service in Manets," Proc. 24th IEEE Int'l Conf. Distributed Computing Systems (ICDCS'04), 2004.

[6] S. Zhong, Y.R. Yang, and J. Chen, "Sprite: A Simple, Cheat-Proof, CreditBased System for Mobile Ad-hoc Networks,"Proc. IEEE INFOCOM, pp. 1987-1997, 2003.
[7] Fudenberg D, Tirole J, “Game theory. MIT Press, Cambridge,” 1991.

[8] Jung S, Hundewale N, Zelikovsky A, "Energy efficiency of load balancing in MANET routing protocols," Sixth International Conference on Software Engineering, Artificial Intelligence, Networking and Parallel/Distributed Computing and First ACIS International Workshop on Self-Assembling Wireless Networks (SNPD/SAWN'05), p 476-483, 2005.

[9] Feeney LM, "An energy consumption model for performance analysis of routing protocols for mobile ad hoc networks," Mobile Netw Appl 6:239249, 2001.

[10] Khouzani MHR, Eshghi S, Sarkar S, Shroff NB, Venkatesh SS, “Optimal energy-aware epidemic routing in DTNs," Proceedings of the thirteenth ACM international symposium on Mobile Ad Hoc Networking and Computing (MobiHoc'12), p 175-182, 2012.

[11] Manam VKC, Mahendran V, Murthy CSR, "Performance modeling of DTN routing with heterogeneous and selfish nodes," Wirel Netw 20(1):2540, 2014.

[12] Wang Y, Singhal M, "On improving the efficiency of truthful routing in MANETs with selfish nodes," Pervasive Mob Comput 3(2007):537-559, 2007.

[13] S. Marti, T. J. Giuli, K. Lai, and M. Baker, "Mitigating routing misbehaviour in mobile ad hoc networks," in Proc. ACM/IEEE Annual International Conference on Mobile Computing and Networking (Mobicom), pp. 255-265, Boston, MA, Aug. 2000.

[14] S. Buchegger and J.-Y. Le Boudec, "Performance analysis of the CONFIDANT protocol (cooperation ofnodes: fairness in dynamic ad-hoc networks)," in Proc. ACM International Symposium on Mobile Ad Hoc Networking and Computing (MobiHoc), pp. 80-91, Lausannae, Switzerland, June 2002.

[15] P. Michiardi and R. Molva, "A game theoretical approach to evaluate cooperation enforcement mechanisms in mobile ad hoc networks," in Proc. IEEE/ACM International Symposium on Modeling and Optimization in Mobile, Ad Hoc, and Wireless Networks (WiOpt), Sophi Antipolis, France, Mar. 2003

[16] E. Altman, A. A. Kherani, P. Michiardi, and R. Molva, "Non-cooperative forwarding in ad hoc networks," in Proc. International Conferences on Networking, vol. 3462, Waterloo, Canada, May 2005.

[17] M. Felegyhazi, J. P. Hubaux, and L. Buttyan, "Nash equilibria of packet forwarding strategies in wireless ad hoc networks," IEEE Trans. Mobile Computing, vol. 5, no. 5, p. 463-476, Apr. 2006.

[18] V. Srinivasan, P. Nuggehalli, C. F. Chiasserini, and R. R. Rao, "Cooperation in wireless ad hoc networks," in Proc. Annual IEEE Conference on Computer Communications (INFOCOM), San Francisco, CA, Mar. 2003.

[19] Srinivasan V, Nuggehalli P, Chiasserini F, Rao RR "Cooperation in wireless adhoc networks," Proc INFOCOM 2003(2):808-817, 2003.

[20] Naserian M, Tepe K, "Dynamic probabilistic forwarding in wireless ad hoc networks based on game theory," 2014 I.E. Vehicular Technology Conference (VTC Spring), 1-5, 2014.

[21] Zhu H, Dusit N, Walid S, Tamer B, Are H, "Game Theory in Wireless and Communication Networks Theory, Models, and Applications," 2011. 